\title{
Performance Measurement and Analysis of Video Conferencing
}

\author{
Rohit \\ Lovely professional University, \\ Jalandhar-Punjab (India)
}

\author{
Himanshu Sharma \\ Lovely professional University, \\ Jalandhar -Punjab (India)
}

\begin{abstract}
Video conference is becoming so popular in today world. People have to go for Distance places for attending meetings but this problem is solved by the videoconferencing. By this one person can communicate with other person using their webcam while remain in the one location. He needs not to go distant place to attend the meetings. It save time as well as money .This paper is for increasing the performance and analyses of the video conferencing. By that the people who are doing video conferencing get the good quality of video conference. This paper tells how to increase the performance by using the SBPN and analysis the video conferencing.
\end{abstract}

Keywords

SBPN,NS2,Video conferencing.

\section{INTRODUCTION}

Video conferencing is also knows as Video teleconference. Because in the Video conference a set of telecommunication technologies which allow us to communicate between Two or more location by simultaneous two way video and audio transmissions.

Due to relatively low cost High capacity broadband telecommunication services in late 1990s, videoconferencing usage has made significant inroads in business, education, medicines and media. Like all long distance communications technologies by reducing the need to travel to bring people together.

In the Video conferencing an application will prepare data and transfer it to transport layer then those data is divided into packets. Then packets is queued, and then sent when it reaches the head of the queue, after all other data before it has been transmitted. This however takes no account of time required to deliver the data. The most important concepts related to service offered by the data (video + audio) network is the quality of the service (QoS). QoS is the capacity of the network to provide better service to data traffic over various network technologies. Some of the primary goal of QoS are bandwidth, controlling and latency and improved loss characteristics. The main Objective of the research is to increase the QoS and make secure the video conferencing. This paper consist the methodology for increase the performance of the video conferencing by increasing the quality of the service. And also contain about the security while doing video conferencing.

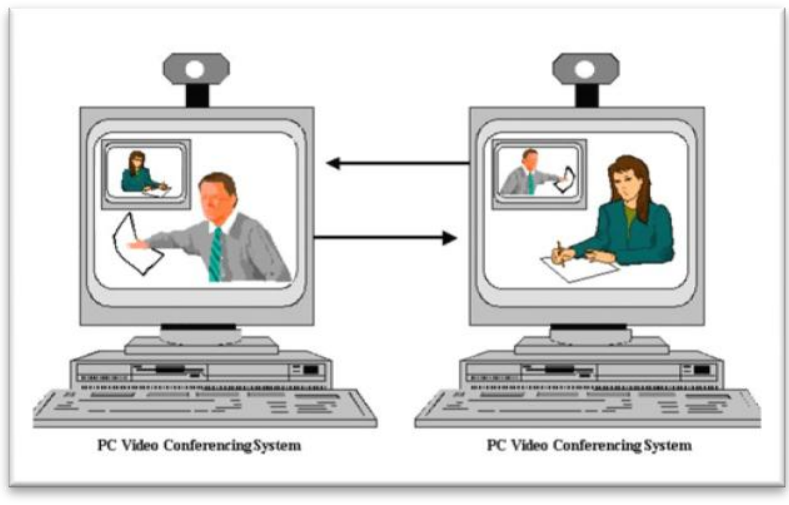

Fig 1. Showing two persons are doing video conferencing.

\section{RELATATED WORKS}

In this section previous work aimed at improving the transmission of video is examined. By nature video generates a considerable amount of network traffic that has strict requirements of the network. As such it is often constrained by the network conditions.

Application-level QoS: improving video conferencing quality through sending the best packet next 25 It is commonly proposed that video codecs should add and remove video layers as network conditions permit .While these codecs match the traffic generated to the available capacity, they do not ensure that the most important data is transmitted first. In a study of multimedia streaming (Guo et al., 2006) it was shown that the median time to change to a lower bit-rate stream was around $4 \mathrm{~s}$.

This indicates that there is scope to improve the user experience during the transition time as it is presumed that a lower bit-rate is being used because of loss. There are also cases where the bit-rate cannot be lowered further and loss occurs. Feng et al. (1999) builds upon changing which video layers are used and proposes a priority queue for delivery of prerecorded video streams where lower priority video layers are only sent after higher priority video layers have been sent. Krasic et al. (2003) investigates storing streaming video in multiple discrete layers which are tailored to the type of client that is requesting the data.

The streaming server, which they call priority-progress, then decides the most appropriate layers for the client and maps these to a priority order within each time segment. At the end of each time segment, if the data has not been transmitted, then the data is discarded by a 'progress regulator'. In Tsaoussidis and Wei (1999) Multimedia Transmission Protocol (MTP) is introduced, which is 
based on TCP Reno but without guaranteed reliability. Packet priority information is sent as a 2 bit field which determines whether packets are retransmitted or not.

This enables MTP to not retransmit data which is of lower priority, as compared to TCP which retransmits all missing data. Time-lined TCP (TLTCP) (Mukherjee and Brecht, 2000) introduces the concept of tracking expiry time for data. TLTCP marks data with an expiry time as we also propose, and discards data if the expiry time is reached before the packet is sent. Unlike the approach in this paper, TLTCP does not take the RTT into consideration but proposes that it is worthwhile to do so. TLTCP is a partially reliable protocol - if the data has not expired and retransmission is requested by the receiver then the data is retransmitted. If the data has expired, a more recent packet is sent instead as the expired packet no longer has value.

\subsection{OUR CONTRIBUTIONS}

We take algorithm for Send Best Packet Next and by sending different average packet size of the data we measurement the performance and analyze the video conferencing. By analyzing the performance when different size packet data is transferred into the video conferencing which is using the Send Best Packet Next there is difference in the reaching data to the other party with whom video conferencing is take place. By this we analyze that which average packet size good for video conferencing and showing the End -to End delay of packets of different size, throughput and packet delay fraction of those packets. The simulator used for this is NS2 (Network Simulator 2).

\section{HOW TO INCREASE QUALITY OF THE SERVICE}

\subsection{SEND BEST PACKET NEXT}

Diagram congestion Control protocol (DCCP) is a session based unreliable transport protocol from datagram. DCCP was used for SBPN as it is a recent protocol designed to accommodate application like multimedia streaming and VoIP. DCCP allow different congestion control methods to be used within the protocol.

\subsubsection{SBPN1}

This algorithm sorts the transmit queue as a priority queue. All higher priority packets get sent prior to any lower priority packets being sent. In Video conference the audio packets have high priority than that of video packets. Those packets have expire time also if the not reached on time the packet get expired. When a packet is due to be sent the expiry time is checked against the current time if

\section{SIMULATION RESULTS}

This section compare the performance and analyse the diffrence when different packet size data is going from SBPN while using video confrencing there are two different average packet size are taken; one average packet size is $64 \mathrm{~kb}$ and other is of $256 \mathrm{~kb}$. expires time is less then this calculated the packets is discarded rather than being sent.

\subsubsection{SBPN2}

The formula for allowed sending rates is:

$\mathbf{X}=\max \left(\min \left(X_{-}\right.\right.$calc, $\left.2 * X \_r e c v\right)$, s/t__mbi $)$

Where

$X \_c a l c=T C P$ throughput equation

$X \_r e c v=$ receive rate since the previous feedback

$s=$ the average packet size

t_mbi is 64 .

This means that in practice the allowed transmit rate falls very quickly when no packets are being transmitted and thus not being received also. This has the impact of reducing the quality of the video conference, particularly during quiet periods. There is an internet draft for TFRC faster restart that partially addresses this issue. To address the issue of reduced transmit rate the SBPN1 algorithm was modified to send an expired packet if it is the only packet in the queue to reduce the likelihood of the transmit rate being reduced.

\subsubsection{RING BUFFERS}

Ring buffer determine whether to a packet at time of queuing for transmission. This combine the two approaches in one algorithm for comparison to see if the combination further improves the number of packets received.

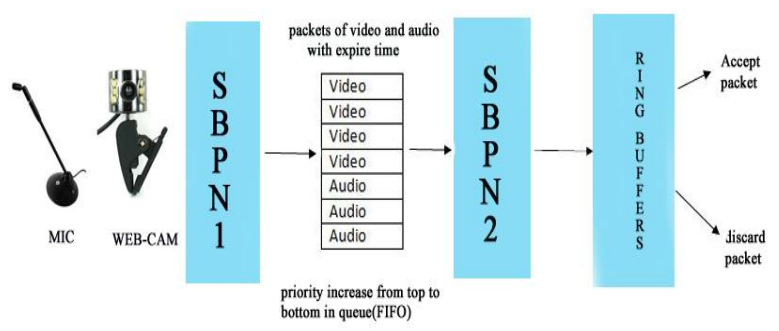

Fig 3.1 Overall Working in SBPN in Video conferencing.

As shown in figure 1 all working of SBPN1, SBPN2 AND RING BUFFERS. First data is coming from webcam and mic then that data is divided into packets by SBPN1 and they are arranged according to their priority audio have higher priority so they are down the queue. And the video packets have less priority then audio so they are above the audio packets. Then the packets are going in SBPN2 there the conditions are checked according to the given formula.And then packets go to the ring buffers there that accept or discard the packets according to conditions. 


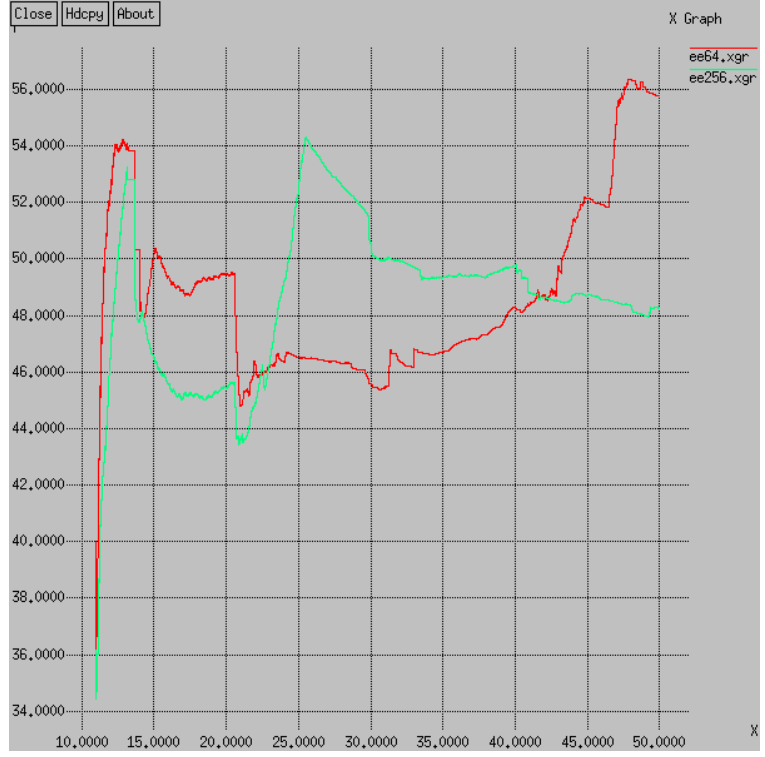

Fig 4.1 . comarison end to end delay of data moving in SBPN of average packet size $256 \mathrm{~kb}$ and $64 \mathrm{~kb}$ while doing video confrencing.

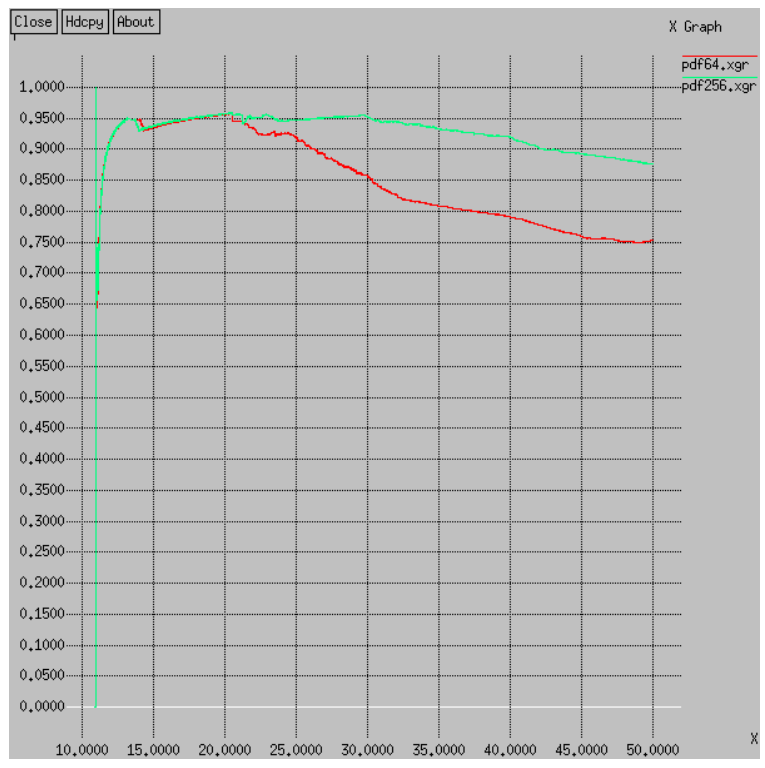

Fig 4.2 - comarison packet delay fraction of data moving in SBPN of average packet size $256 \mathrm{~kb}$ and 64 kb while doing video confrencing.

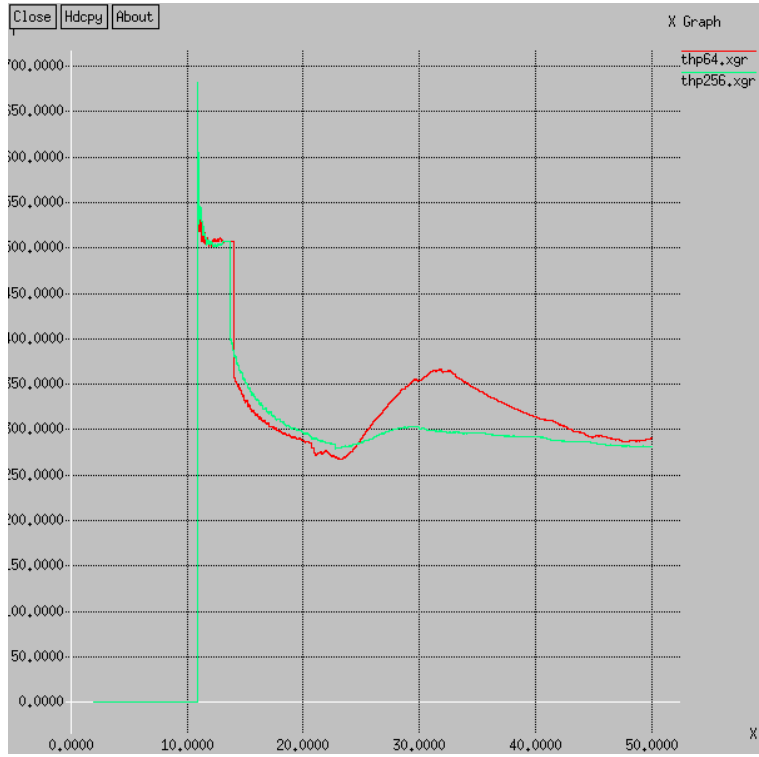

Fig 4.3 . comarison throughput of data moving in SBPN of average packet size $256 \mathrm{~kb}$ and $64 \mathrm{~kb}$ while doing video confrencing.

\section{CONCLUSION AND FUTURE SCOPE}

By using the Send best packet next we can increase the QoS and make the video conferencing of good quality and performance. And analysis that which average packet size give batter performance. As in given set there are two different sizes are taken average size 64 and 128. As shown in Figure 3, 4, 5 the average packet size 64 is batter to use then that of 256 because it takes less time to reach to the other person.

We are only work on one algorithm of Send best packet next there may be possibility that some other algorithm perform batter in send best packet next then that of we used in our SBPN.

We are testing this algorithm only with two average packet sizes but there may be possibility that this perform batter with some other average packet size value and that average packet size can be used for best performance of video conferencing.

\section{ACKNOWLEDGMENTS}

I thank my parents and friends for all of the love, support, and encouragement they have provided me throughout my life. I am forever grateful for the sacrifices they made to give me the wonderful opportunities that I have had.

\section{REFERENCES}

[1] Amer, P.D., Chassot, C., Connolly, T.J., Diaz, M. and Conrad, P. 'Partial-order transport service for multimedia and other applications', IEEE/ACM Transactions on Networking (1994).

[2] Balk, A., Maggiorini, D., Gerla, M. and Sanadidi, M.Y. 'Adaptive MPEG-4 video streaming with bandwidth estimation', Proceedings of the Quality of Service in Multiservice IP Network (2003).

[3] Brakmo, L.S. and Peterson, L.L. (1995) 'TCP vegas: end to end congestion avoidance on a global internet', IEEE Journal on Selected Areas in Communications. 
[4] http://hive1.hive.packetizer.com/users/h323forum/pap ers/vcon_031113.eng.wp.Firewalls_and_Proxy_Serve rs.pdf

[5] Frost and Sullivan "Delivering on the promise of easy to use,secure and inexpensive video conferencing in an environment",(2012)

[6] Mario Baldi, "End-to-End Delay of Videoconferencing Over Packet-Switched Networks",IEEE,(1998)
[7] N.Celanfroni,F.Davoil,E.Ferro,S.Zappatore,"An Experiment study of the quality of the service of video Encoded Sequences over an Emilated Rain faded satellite Channel",IEEE,

[8] Rohit,Himanshu Sharma,"Methodology for Improving performance of video conferencing with additional security”. IJCMR .(2012) 